\section{Experimental Analysis of Dynamic Interaction Between a Centrifugal Compressor and Its Casing}

\begin{abstract}
In turbomachinery, one way to improve aerodynamic performance and reduce fuel consumption consists of minimizing the clearance between rotor and casing. Yet, the probability of contact is increased and this may lead in some specific conditions to a large and even unstable excitation on the impeller and stator. To achieve better understanding of the dynamic behavior occurring during the blade-to-casing contact, many numerical studies have been conducted but only a few experiments have been reported in the literature thus far. The interaction experiment reported in this paper involves a low-pressure, rotating centrifugal compressor and its casing tested in a vacuum chamber. Contact is initiated by introducing a gap near zero, and certain events with significant dynamic levels are observed during the run-up. Measurements are performed using strain gauges on both the rotating and stationary parts and a scanning laser Doppler vibrometer on the stator. This research focuses on an analysis of the recorded data. Time series data are also analyzed by means of standard signal processing and a full spectrum analysis in order to identify the direction of traveling wave propagation on the two structures as well as nodal diameters and frequencies. The dynamic response of structures is accompanied by variations in other physical parameters such as temperature, static deformed shapes, speed, and torque. A wearing pattern is evaluated following the contact experiments. The spectral content of response is dominated by frequency modes excited by rotating speed harmonics as well as by sidebands due to inherent system nonlinearity.
\end{abstract}

Keywords: blade-casing contact, short-time Fourier transform, traveling waves, full spectrum, thermal effect, abradable coatings, wear

\author{
P. Almeida \\ École Centrale de Lyon,
Laboratoire de Tribologie et Dynamique \\ des Systèmes, \\ 36, avenue Guy de Collongue, \\ Ecully Cedex 69134, France \\ e-mail: patricio.almeida@ec-lyon.fr
}

C. Gibert

École Centrale de Lyon, Laboratoire de Tribologie et Dynamique des Systèmes,

36, avenue Guy de Collongue, Ecully Cedex 69134, France

F. Thouverez

École Centrale de Lyon, Laboratoire de Tribologie et Dynamique des Systèmes,

36, avenue Guy de Collongue, Ecully Cedex 69134, France

X. Leblanc

École Centrale de Lyon, Laboratoire de Tribologie et Dynamique des Systèmes,

36, avenue Guy de Collongue, Ecully Cedex 69134, France

J.-P. Ousty

Turbomeca-Safran Group, Bordes Cedex 64511, France

\section{Introduction}

In axial and centrifugal compressors, minimizing the clearance between the blade tips of the impeller and its surrounding casing increases aerodynamic efficiency but also the probability of contacts. An energy exchange is then produced between the two structures, leading to forced excitation of the natural modes or even modal interaction phenomena $[1,2]$, which is a dynamical instability. A detailed analysis of physical phenomena presents during bladecasing contacts was presented in Ref. [3]. Numerical studies based on finite element models were used to predict the vibratory behaviors associated with contacts on axial [4,5] and centrifugal compressors [6]. Given the complexity of the phenomena, experimental setups were developed to get a better understanding on the physics involved during contacts [7-10]. To our knowledge, blade-casing contact experiments were only studied with axial compressors.

This work focuses on the study of the dynamic and thermomechanical behavior resulting from blade-to-casing interaction between a real centrifugal compressor and its casing. The contact experiment is performed within a controlled and instrumented environment. The test bench uses the following simplification: vacuum and no coupling with the shaft dynamic. The main purpose of this paper is the building of a likely scenario to explain the various phenomena present during the test and the creation of a database for subsequent comparison with numerical simulation results.
The paper is organized as follows. First, the experimental setup is described, focusing in the instrumentation and the modal analysis of both structures. Then, the recorded data are presented and analyzed using signal processing tools adapted to testing analysis.

\section{Experimental Setup}

2.1 General Description. An overview of the test rig is shown in Fig. 1. The system consists of a stiff shaft supported by a preloaded precision roller and ball bearings driven by an electric motor that provides a rotational speed in the range of $0-6000 \mathrm{rpm}$. An actual low-pressure centrifugal compressor with ten main blades and ten secondary blades is thermally fitted to the shaft at the rear bore. The shaft of the test rig has been designed to avoid any critical

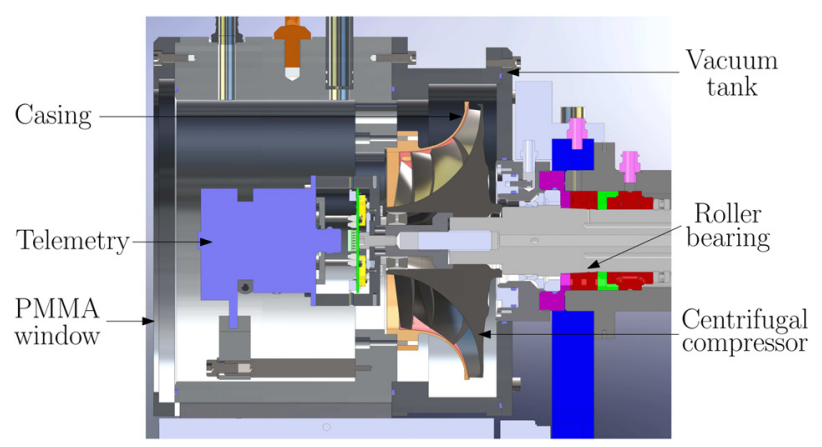

Fig. 1 Test rig general view 
rotor speed within the speed range. A real casing is clamped near the leading edge inside a stiff vacuum tank, thus providing optical access to the front (the polymethyl methacrylate window). This setup allows for laser vibrometer measurements on both the stationary and rotating parts. The casing was redesigned to produce modal interactions within the test bench speed range. The signal from the strain gauges on the rotating part is transmitted via an inductive telemetry system. The vacuum tank, in turn, has been mounted on a ball rail table; a linear motor is introduced to adjust the gap between the two structures by moving the stator along the impeller axis.

Test conditions were monitored using accelerometers, thermocouples, strain gauges, a torquemeter, and an angular position sensor. These tests were performed at room temperature and under vacuum conditions.

2.2 Instrumentation. Figure 2 shows the location of strain gauges and thermocouples on both structures. The impeller has ten strain gauges on the leading edge, three on the blade tip, and three on the trailing edge of all three main and secondary blades. Gauges used on the impeller feature a thermal compensation for titanium (gauge types EA-05-062AQ-350 and CEA-05-124AQ350 ). The placement of strain gauges was carefully chosen, as described in Ref. [11].

On the casing, eight strain gauges were bonded onto the trailing edge and another four onto the middle chord. The stator's gauges offer a thermal compensation for steel (gauge type EA-06062AQ-350), and their conditioning is provided by a half-bridge powered by $5 \mathrm{~V}$ DC. Eight thermocouples of type K were used to measure the casing temperature; these were placed at an angle

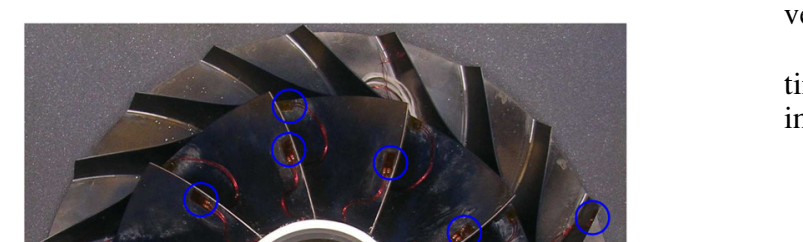

between the strain gauges and equally distributed along the external face of the casing near the trailing edge.

Modal characterization was carried out with two embedded lead zirconate titanate (PZT) piezoelectric plates working in $\mathrm{d} 31$ mode for the centrifugal compressor $[11,12]$ and a piezoelectric stack on the stator. The speed and torque were measured (or derived), respectively, by a shaft position encoder and a strain gauge torquemeter. Wear was measured using a high resolution linear variable differential transformer (LVDT) sensor affixed to the centrifugal compressor (see Fig. 3). The LVDT is slowly rotated around the casing in order to measure the wearing pattern on the abradable coating after the runs.

2.3 Modal Analysis. The modal parameters of both structures will be helpful in analyzing the response during interaction. This modal analysis is conducted by a standard curve-fitting of frequency response functions (FRFs) measured separately on the structures. Sample frequency ranges containing the initial family of modes are given in Figs. 4 and 5. Frequencies are normalized with respect to the mean frequency of the first family of modes of the two structures at rest.

A modal analysis has been performed for the first four mode families on the impeller (Fig. 6) and for the two mode families on the casing (Fig. 7). Figure 8 illustrates one modal shape of the casing measured along the axial direction using scanning laser Doppler vibrometer (SLDV) for one mode of the first family. The natural frequencies, damping factors, and mode shapes, along with their nodal diameter numbers, have been extracted from measurement data. The modal analysis was completed under various thermal conditions and has revealed a decreasing frequency rate versus temperature [11].

Modal parameters allow us to define the structural dynamic time scale; this time constant may be determined using the following logarithmic decrement:

$$
T_{m}=\frac{m \ln (10)}{\xi \omega}
$$

where $T_{m}$ is the time required for the amplitude to decrease by $m$ orders of magnitude, $\xi$ the damping ratio, and $\omega$ the natural frequency of the selected mode. This time scale will be related to duration of bursts in the following.

It has been found that the casing damping ratios are one or two orders higher than those of the impeller. Let us recall that the damping ratios of the impeller are very sensitive to pressure, as shown in Ref. [13], whereas such is not the case for the stator.

The Campbell diagram in Fig. 9 has been established using previously identified frequencies at rest. Please note that the stress stiffening is negligible within the speed range [11]. So, the Campbell diagram could be estimated directly from modal tests

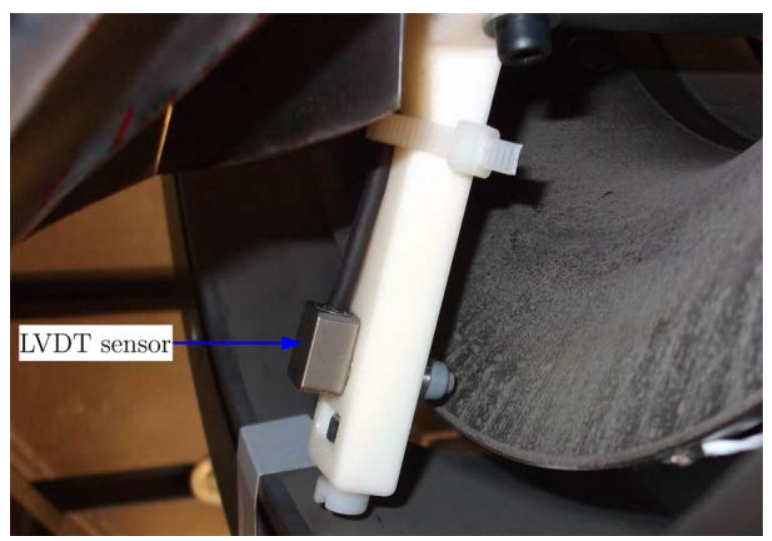

Fig. 3 LVDT sensor fixed on the centrifugal compressor

Fig. 2 Instrumentation installed on both structures 


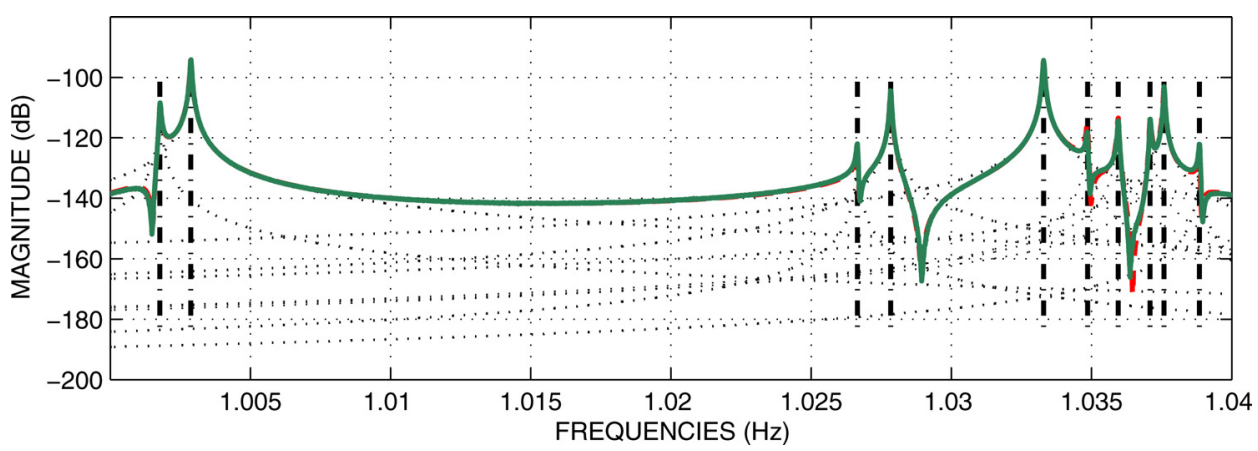

Fig. 4 Typical impeller FRF: measured FRF (- - ), synthesized FRF (-), modal participation of each mode (thin curves)

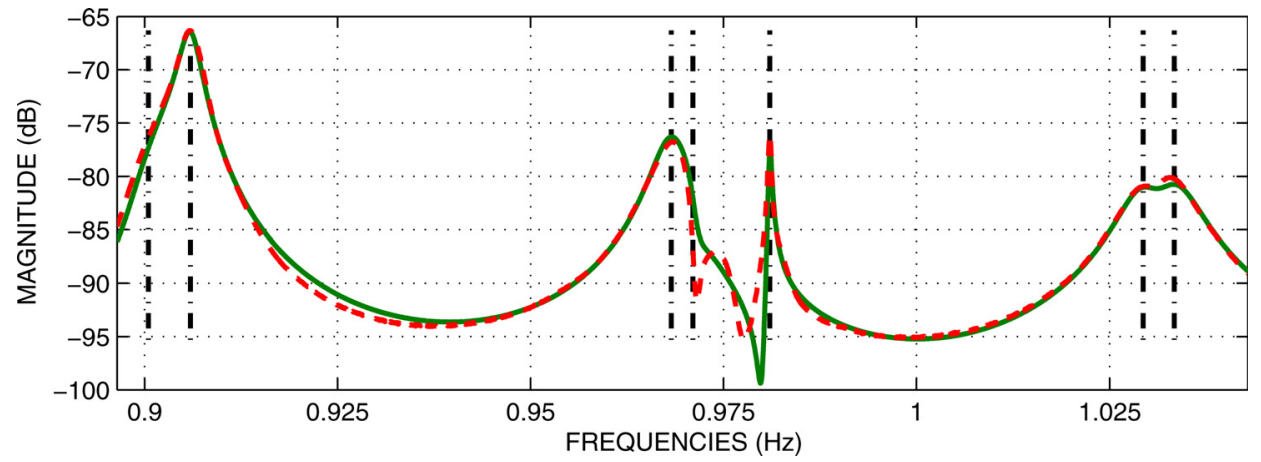

Fig. 5 Typical casing FRF: measured FRF (- - ), synthesized FRF (-)

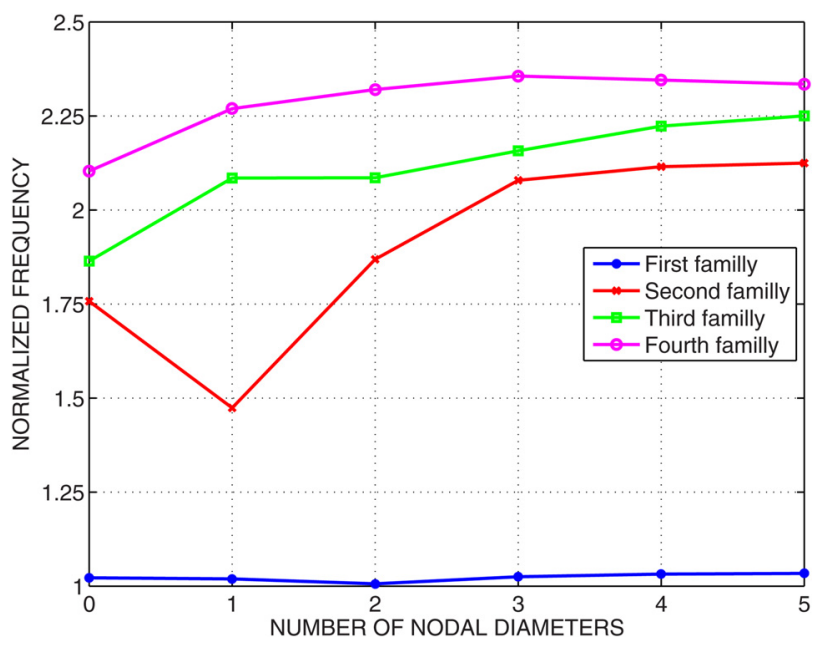

Fig. 6 Family modes of the impeller

performed at rest. In the experimental diagram, frequencies appear as separate pairs for some modes due to mistuning. Moreover, as mentioned by Schmiechen [2], this mistuning has a stabilizing effect on the coupled system dynamics with respect to modal interaction phenomena. This feature is unavoidable for actual structures and should be taken into account in numerical predictions.

2.4 Testing Objectives. The main purpose of this paper is to study the dynamic and thermomechanical behavior of an impeller and a casing during contact. The contact can be engaged either dynamically by modal interaction, either by reducing bladecasing clearance at standstill and then by forcing contact through use of the centrifugal effect [3]. The contact is initiated here by

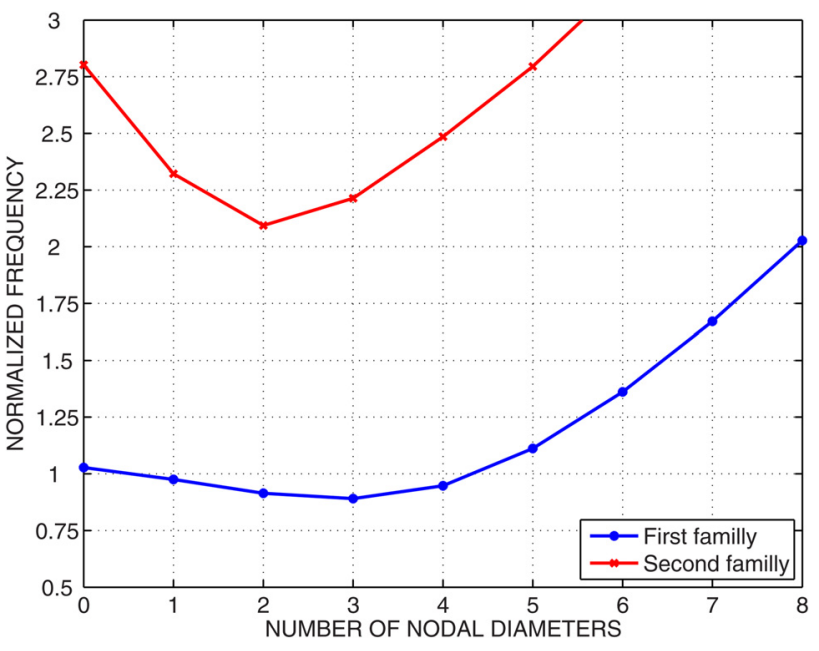

Fig. 7 Family modes of the casing

closing the gap between the structures. The two parts are precisely aligned and centered with respect to one another by a radial clearance at the leading edge. Contact is likely to occur at the trailing edges, once the axial gap is closed. The radial gap between the two structures at the leading edge is kept when the rotor is moved axially. Another purpose is to study the dynamic behavior, particularly the expected modal interaction case when the natural impeller frequency coincides with the natural casing frequency, as expressed within the same frame [2].

$$
\left|\Omega-\frac{\omega_{\mathrm{r}}}{n_{d}}\right| \simeq \frac{\omega_{\mathrm{s}}}{n_{d}}
$$




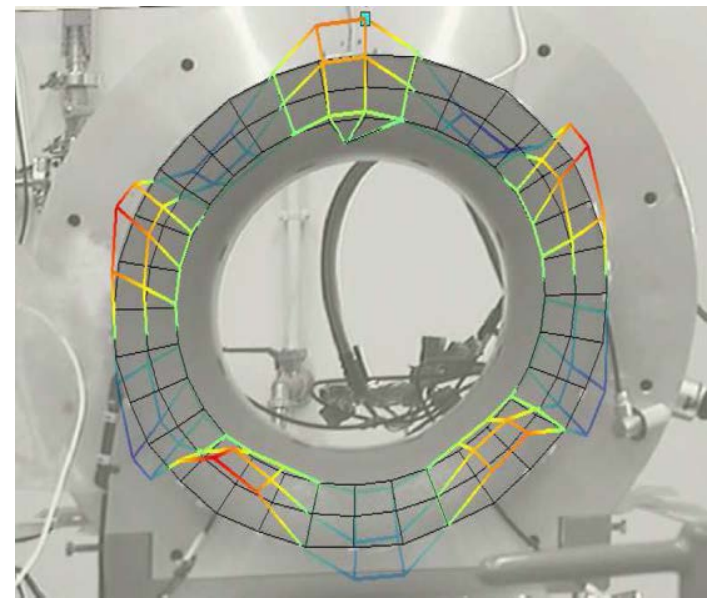

Fig. 8 Example of an operational deflection shape closed to a resonance with 5ND

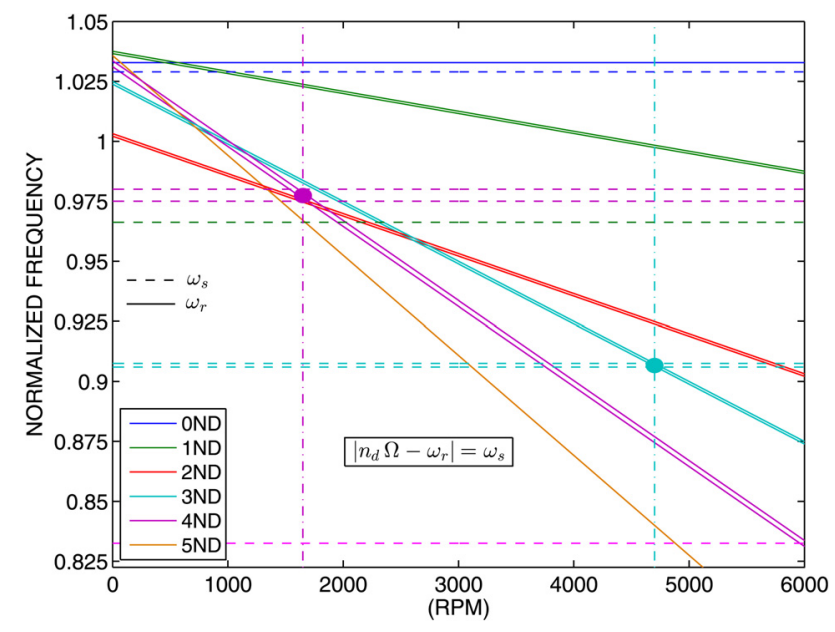

Fig. 9 Campbell diagram in stationary frame

with $\Omega$ the rotating speed, $\omega_{\mathrm{r}}$ the natural impeller frequency, $\omega_{\mathrm{s}}$ the natural casing frequency, and $n_{d}$ the nodal diameter. From literature, energy can only be exchanged in the case of two modes with the same number of diameters. It is admitted for this case that Eq. (2) is a necessary condition for dynamic instability.

Modal interaction may be predicted by means of the Campbell diagram pictured in Fig. 9 [3]. Two critical velocities were found within the test rig speed range, where $\Omega_{\mathrm{c}}=\left(\omega_{\mathrm{r}}-\omega_{\mathrm{s}}\right) / n_{d}$. The target modes were: $4 \mathrm{ND}$ at $1560 \mathrm{rpm}$ and $3 \mathrm{ND}$ at $4720 \mathrm{rpm}$. The speed condition applied to the presented herein corresponds to a constant value below the critical speed for the 4ND $\left(\Omega<\Omega_{\mathrm{c}}\right)$.

2.5 Test Description. The contacting process was performed in several steps. First, the impeller was rotating in the rig (under vacuum conditions at a selected speed), with an initial gap that avoided any contact until stabilization of the bearings temperature. The modal parameters of the two structures were then identified using strain gauges and SLDV. The target frequency modes were used to establish critical velocities $\Omega_{\mathrm{c}}$. The test rig was then shut off to adjust the axial position between the centrifugal compressor and the casing. The initial gap was set close to zero. The presence of contact was detected using SLDV on the casing. SLDV was then set at its maximum sensitivity level. The level of sound perception when the blade touches the abradable coating and a visual test using ink applied to the blade tips have served to confirm the contact. However, it was noticed that this contact was not maintained throughout an entire revolution due to the inevitable geometric imperfections. Vibration of blades was monitored during test and the rig stop when a given threshold was reached. This several observations showed that the initial contact is probably very light.

Next, the recording step begun, and the speed was set at the desired value (constant speed).

\section{Time Analysis}

In this section, we will present in detail one of several runs with this test rig looking at time histories recorded from the different sensors.

3.1 Dynamic Response. During the test, the dynamic response of both the impeller and casing displayed eight transient events (bursts of vibration), characterized by a simultaneous increase in amplitude on both structures (see Fig. 10). These transient events will be called bursts of vibration or shorter "bursts" in this paper. The final burst of vibration had the highest amplitude, at, respectively, two and five times greater than the others on the impeller and casing. The duration of each burst was on the order of $T_{m}$-see Eq. (1)—and associated with dynamical transient responses of structures. The burst was spaced in time by intervals of one or two orders of magnitude greater than $T_{m}$.

Speed and torque signal fluctuations were noticeable whenever the bursts occurred, as illustrated in Fig. 11. This variation was attributed to resistant torque due to tangential forces acting between the two structures.

3.2 Thermal Response. Figure 12 shows the temperature history during the test. An eight-step thermal evolution is visible, with the transition from one step to the next being initiated by a burst. The maximum temperature was obtained with thermocouples TC1 and TC8. At the fourth burst, TC1 reached $56^{\circ} \mathrm{C}$ and the temperature decreased afterward. The decay of temperature measured after a burst matches with the free thermal response of a numerical conduction model.

The temperature profile of TC8 revealed a similar behavior to the other thermocouples until the fifth burst. Ultimately, after the sixth burst, the temperature peak was reached at $180^{\circ} \mathrm{C}$.

During bursts, whenever the vibration level increased, the thermal behavior tended to become uniform on the casing (probably by heat conduction). At the highest burst, the thermal field appeared to be more uniform, as pictured in Fig. 12, whereas it appeared localized during previous bursts. It is believed that a higher vibration level tends to increase the contact area on the stator despite the geometrical imperfectness mentioned before. Although a decay of temperature was seen after each burst, note that cumulative effect produced a general warming of the stator.

3.3 Quasi-Static Part of the Response. The quasi-static part of the strain gauge response on the casing is shown in Fig. 13. This output was obtained by applying a numerical low-pass frequency filter to the signals. The frequency cut was approx. $0.5 \%$ of the first impeller frequency. The maximum quasi-static deformation was measured by gauges located between the thermocouples measuring the highest temperature peaks. The quasi-static deformation of the casing is localized, and this localization is indeed due to geometric imperfections resulting in a nonuniform gap. The quasi-static analysis combined with the thermal analysis is used to indicate zones where the contact is supposed to occur. The first zone was identified between gauges R_01 and R_02 (Fig. 2) for the first and third bursts; the peak temperature at thermocouple TC1 confirms this assertion. Let us recall that the thermocouples were placed in between the strain gauges. During the fourth burst, strain gauge R_01 measured the highest quasi-static response; moreover, TC1 yielded the highest temperature. This finding was interpreted as a displacement of the contact area near the zone where strain gauge R_01 was located. This could be attributed to thermal expansion or wear. Then, gauges R_01 and 

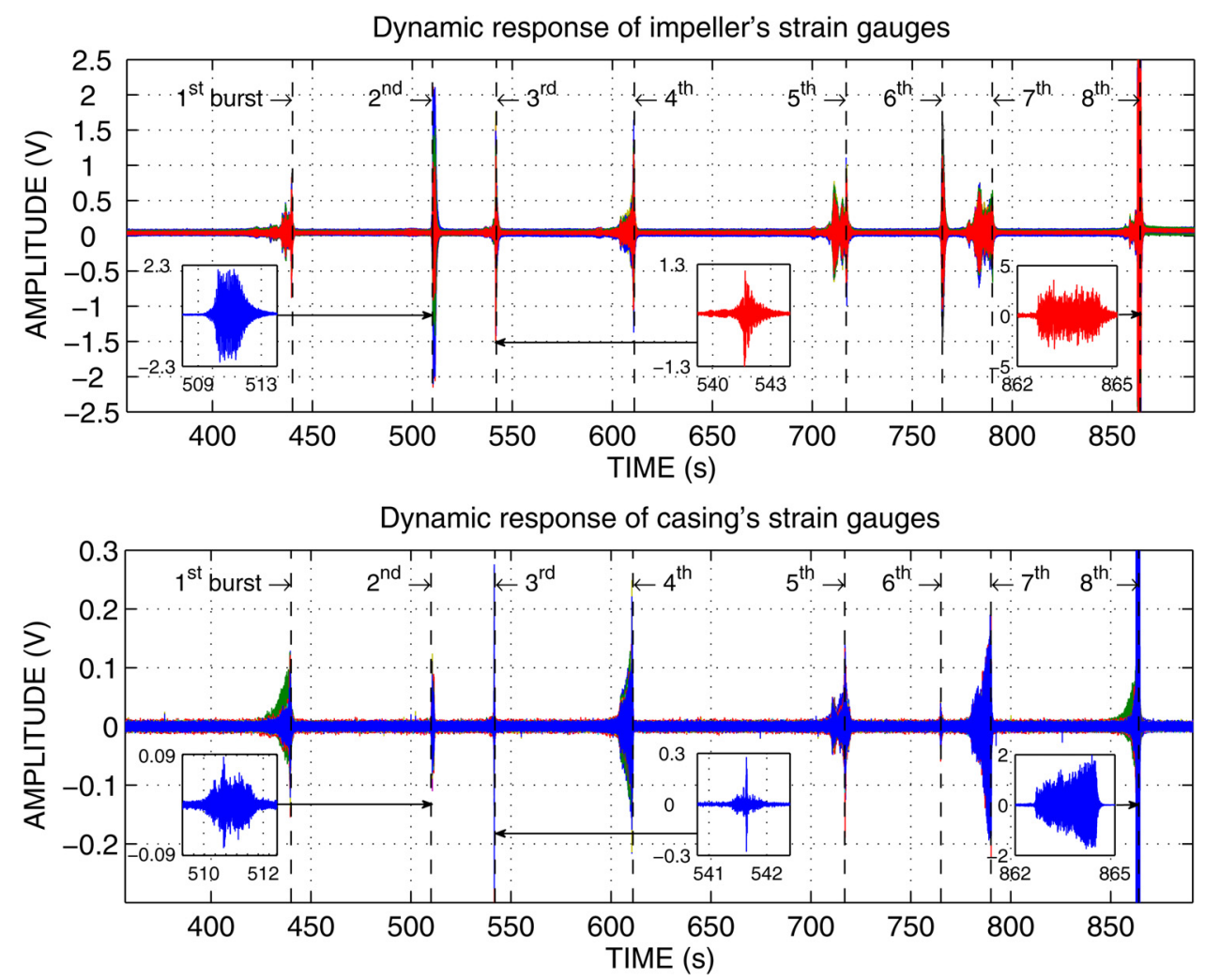

Fig. 10 Dynamic response of the impeller (top) and the casing (bottom)

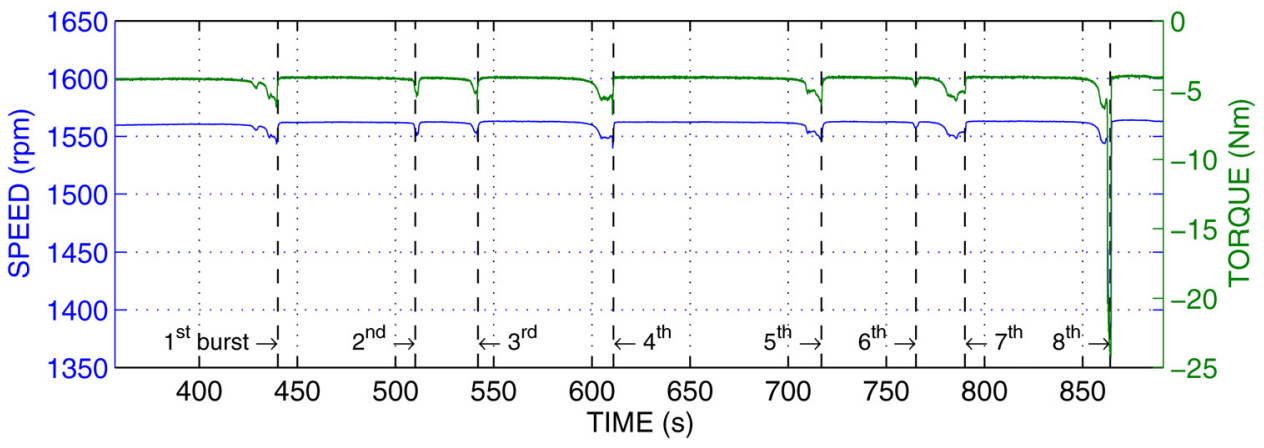

Fig. 11 Speed and torque time history

R_08 recorded the highest quasi-static response, while TC8 showed the highest temperature at the seventh burst. A similar situation can be detected near TC8. During the final burst, all strain gauges seemed to lie at the same quasi-static level and temperature was also increasing uniformly on the casing. This result might be interpreted as the uniform closure of the gap due to strong vibration.

Using the finite element model of the casing, it can be shown that a positive strain at the gauges corresponds to a static deflection in the direction opposite the impeller. The instantaneous time when the maximum quasi-static deformation occurred corresponds to the instantaneous time of the maximum dynamic part. When the dynamic part vanishes, the quasi-static part returns to equilibrium contrary to the thermal behavior. The width of peaks observed on the quasi-static part is on the order of $T_{m}$, see Eq. (1), which may be interpreted as a coupling between dynamic and static equilibrium positions of the stator due to contact nonlinearity.

\section{Frequency Analysis}

4.1 Spectral Analysis Tools. Two spectral analysis tools will be applied to the following signals. A short-time Fourier transform (STFT) with a temporal window provides the spectrogram of each measurement channel versus time [14]. The temporal window has been chosen in order to provide a satisfactory tradeoff between time and frequency resolutions [15].

The other tool used in this paper is based on a two-dimensional discrete Fourier transform (2D DFT) applied to a set of sensors equally spaced on both structures [13]. Ten strain gauges placed on the impeller leading edge and eight strain gauges bonded onto the casing trailing edge were used. For each frequency, the 2D DFT yielded the spatial decomposition arranged in nodal diameters. The full spectrum analysis allows identifying the direction of the rotating wave for each nodal diameter [16]. A negative nodal diameter was interpreted as a backward traveling wave. A corresponding negative and positive nodal diameter with equal amplitudes corresponds to a standing wave. Full spectrum analysis was motivated by the fact that different modal coincidences on the Campbell diagram were characterized by different combination of wave propagation direction (cf. backward/forward) [3]. This could be used to verify the assumption given by some references $[4,6]$ that the contact forces always produce backward traveling modes on the bladed disks. This analysis could be performed at several 

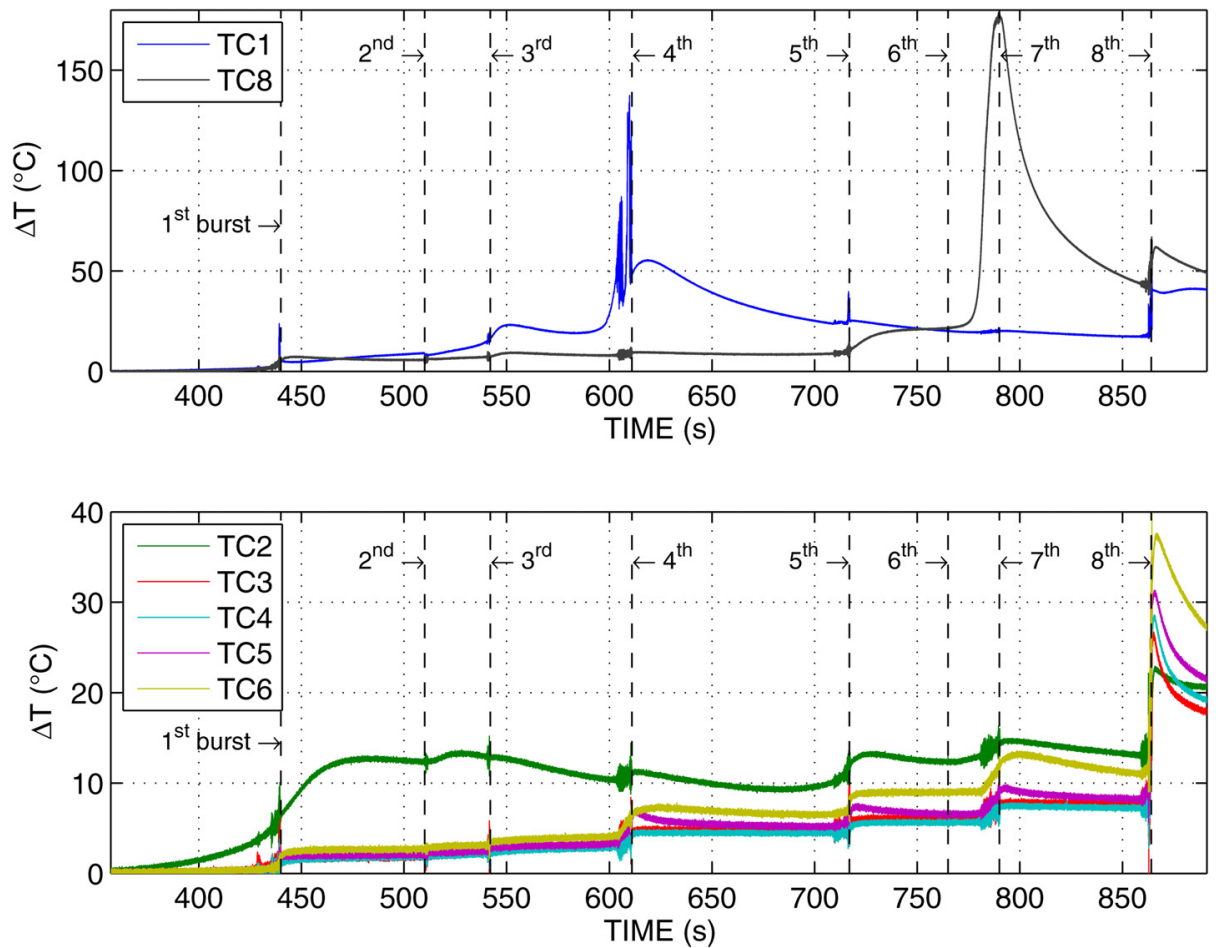

Fig. 12 Temperature history on the stator

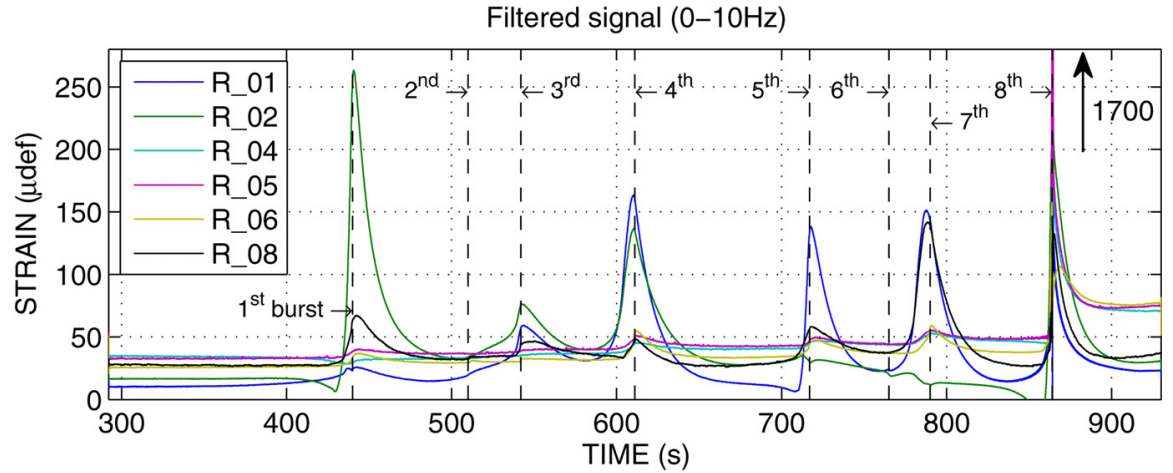

Fig.13 Quasi-static response of the casing

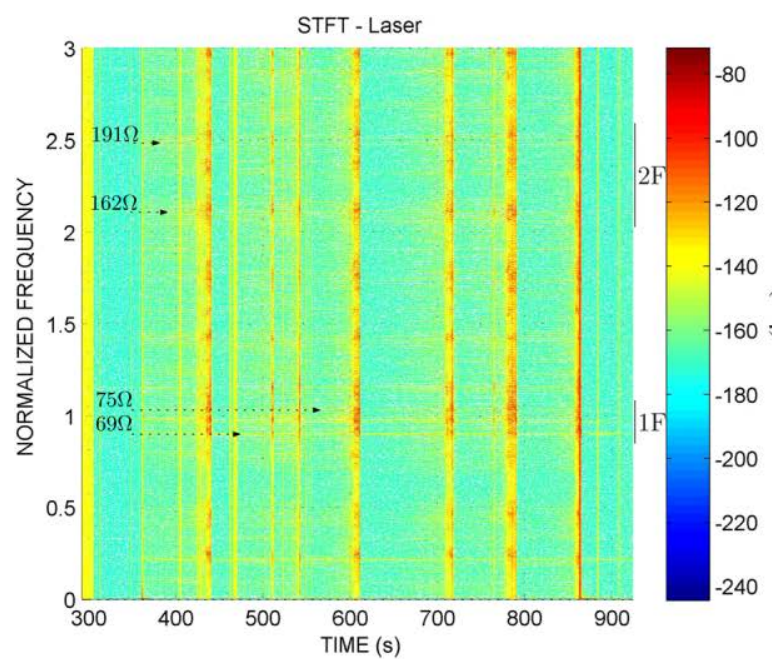

(a)

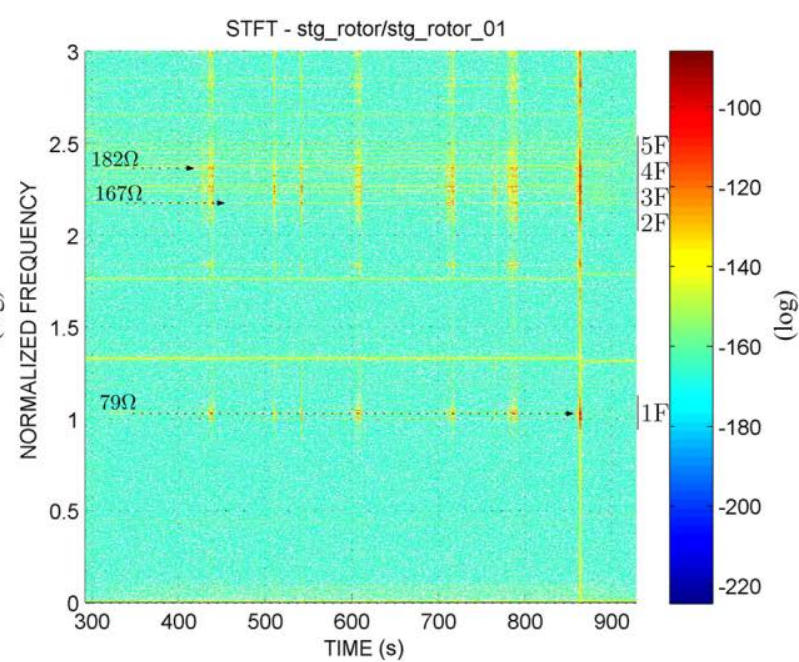

(b)

Fig. 14 STFT with rotating speed harmonics (a) casing and (b) impeller 


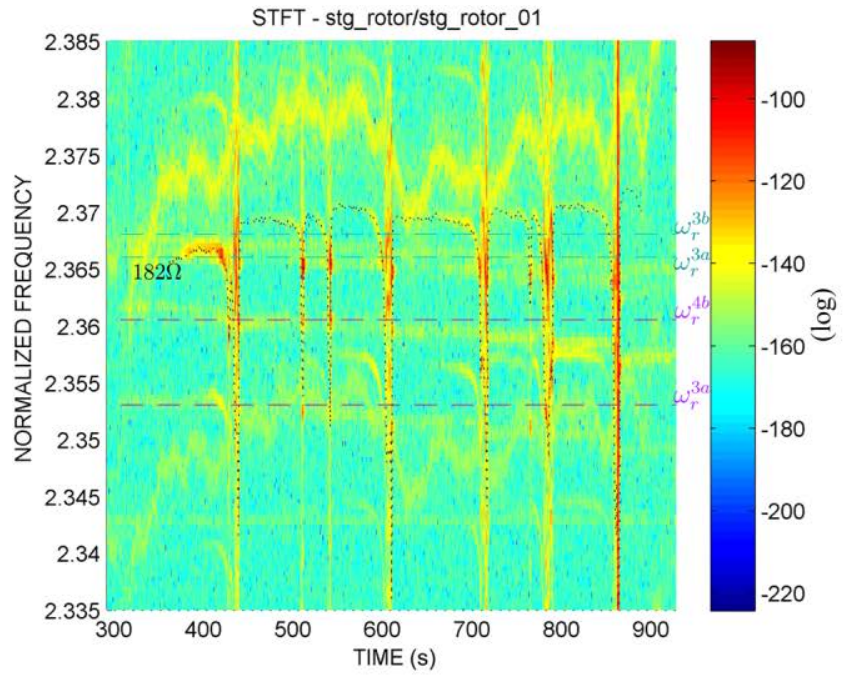

(a)

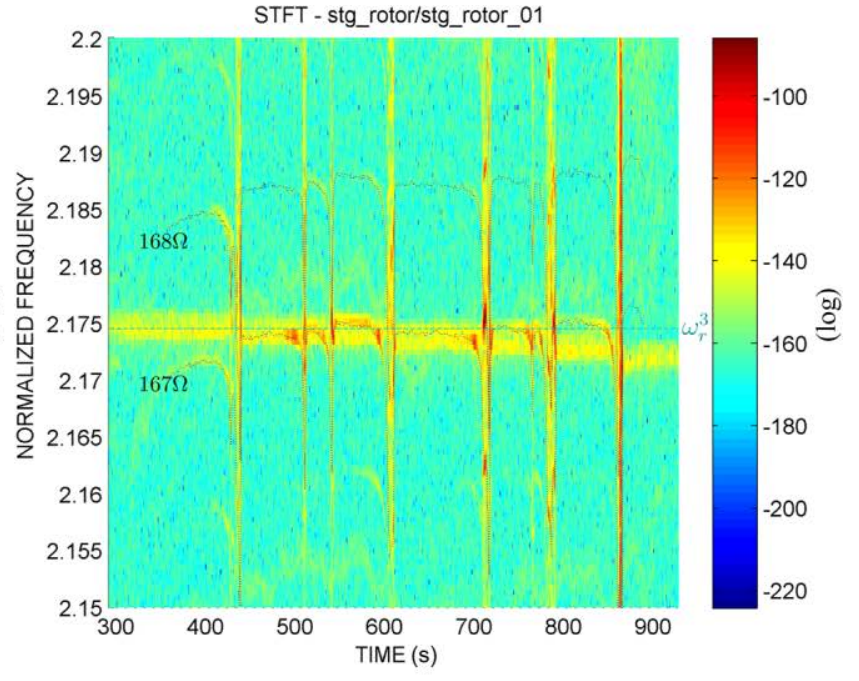

(b)

Fig. 15 STFT of the impeller: initiation of bursts $(a)$ initiation of the first burst and $(b)$ initiation of other bursts
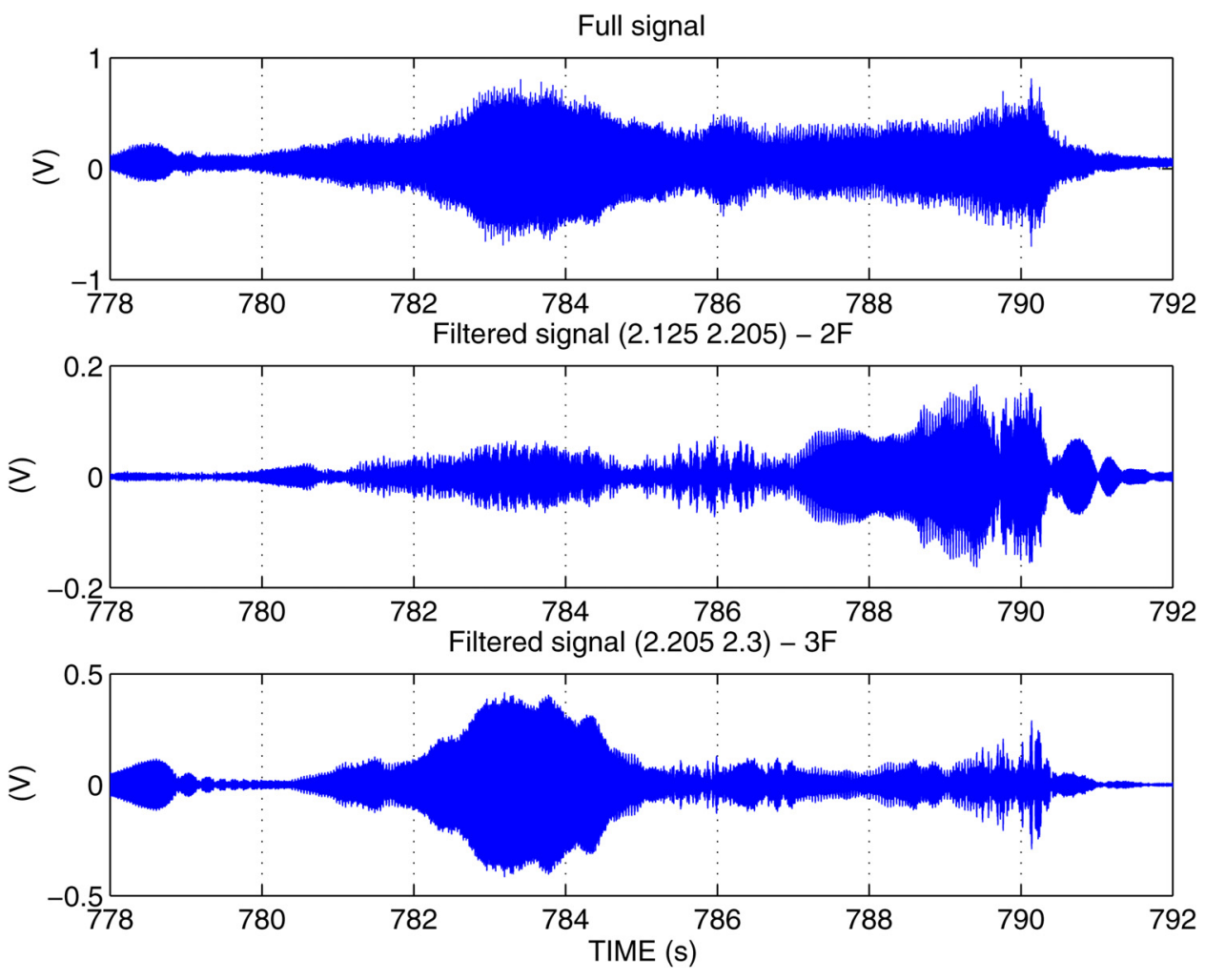

Fig. 16 Family contributions at seventh burst

different times over the entire recorded signal. Let us note that the ten measurement points on the wheel provide sufficient spatial sampling to identify five different diameters. On the other hand, the number of sensors (8) on the stator limits the analysis to four diameters. In that case, the finite element method could help the identification of modal diameter.

4.2 Results and Discussions. The STFT was performed using the signal from a leading edge gauge on the centrifugal compressor and the SLDV signal on the casing. Figure 14 shows the STFT of the selected sensors in the frequency range (0-3). In this same figure, the rotating speed harmonics with the highest amplitudes during the test can easily be observed.
This analysis was conducted for all measurement channels on both structures. In Fig. 15, the natural frequencies of the impeller are plotted on the spectrogram zoomed on $3 \mathrm{~F}$ and $4 \mathrm{~F}$. The strong contributions of the third and fourth family modes of the impeller are readily apparent, with such contributions triggering burst initiation. Prior to the first burst, harmonic 182 of the rotating speed $(182 \Omega)$ coincides with the fourth family mode of the impeller and with 3ND (see Fig. 15(a)). It seems that modal excitation by rotating speed harmonics increases vibration on both structures, thus leading to a burst. For the other bursts, the third family mode of the impeller with 3ND is excited by harmonic 167 of the rotating speed $(167 \Omega)$, as depicted in Fig. 15(b). Note that as the speed decreases, one mode may be excited successively by two 


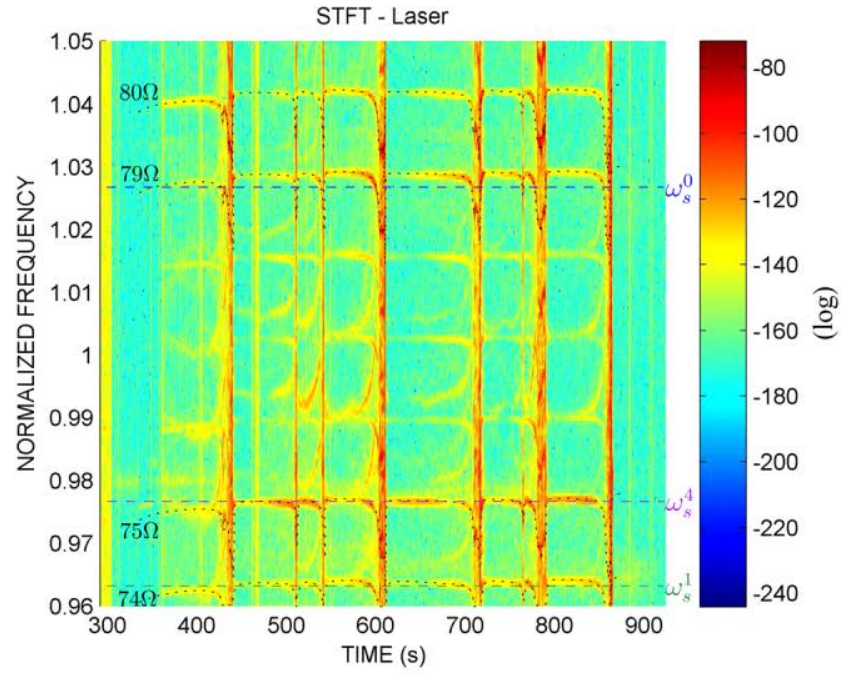

(a)

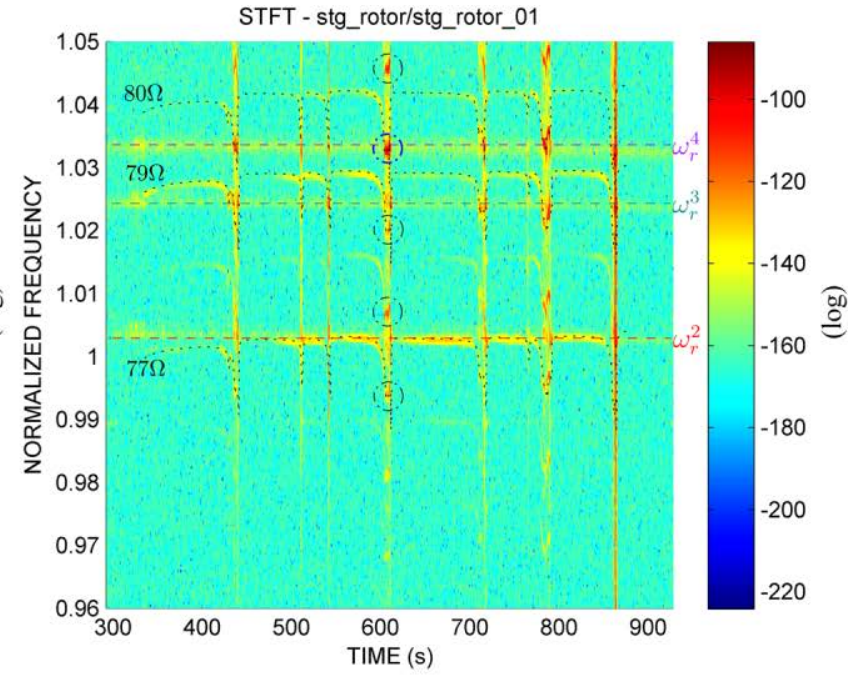

(b)

Fig. 17 STFT spectrum of the first family modes of both structures (a) casing and $(b)$ impeller

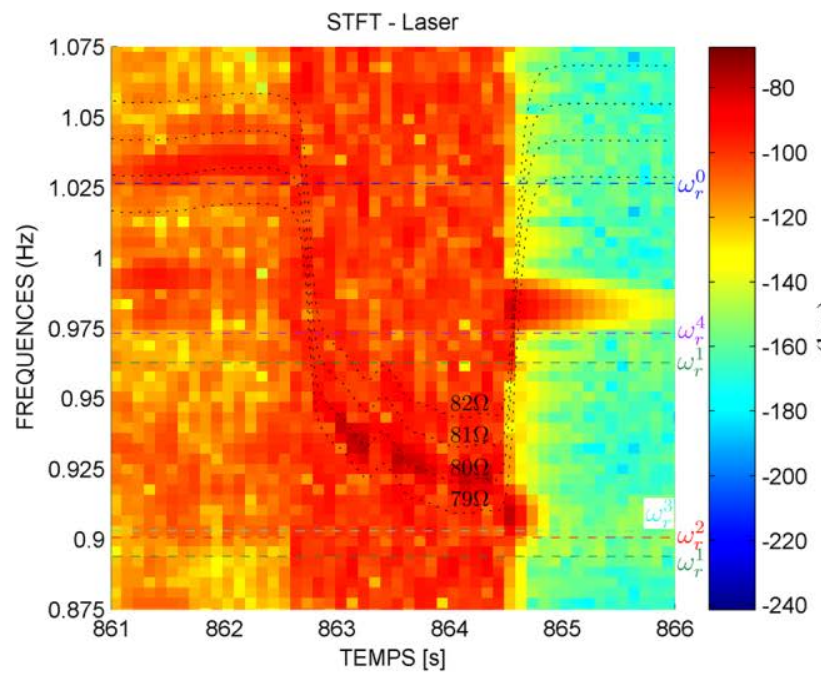

(a)

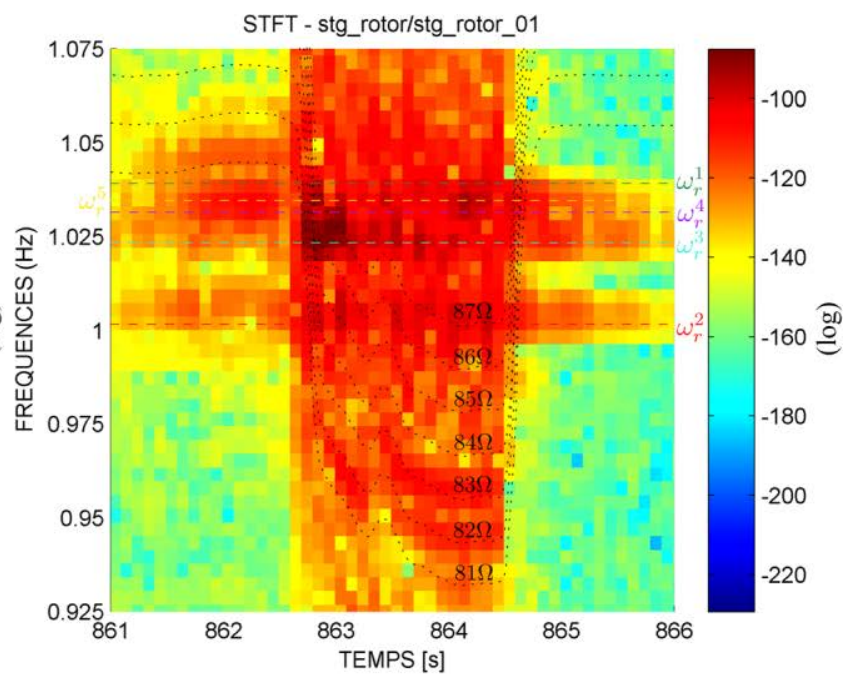

(b)

Fig. 18 STFT spectrum zoomed on the eighth burst (a) casing and $(b)$ impeller

or more passing rotating speed harmonics. Figure $15(b)$ shows the excitation of the third family mode of the impeller with 3ND by harmonic 168 of the rotating speed $(168 \Omega)$ during the fifth burst.

Please note that the thermal influence on frequencies is clearly highlighted by the evolution of the yellow trace which correspond to the natural impeller frequency $\left(\omega_{\mathrm{r}}^{3}\right)$. Indeed, one can see that the frequency decreases with time as the temperature increases.

During the seventh burst, two family modes of the impeller (i.e., the second and third families) belonging to two different frequency ranges were excited at two different times, as shown in Fig. 16. Note that, as it has been shown above, the nodal diameters from 0ND to 2ND does not participate in the response, which make possible to use frequency filters in order to identify the family modes excited during the response.

The spectrogram analysis displayed in Fig. 17 was performed around the first family modes of both structures. The horizontal dashed lines and dotted lines indicate, respectively, the natural frequencies and rotating speed harmonics. The STFT of a casing channel-given in Fig. 17(a)-shows the coincidence between harmonic 75 of the rotating speed $(75 \Omega)$ and mode with 4ND $\left(\omega_{\mathrm{s}, \mathrm{S}}^{4}=75 \Omega_{\mathrm{c}}\right.$ at the stationary frame ${ }^{1}$.

This coincidence leads to a sufficiently high excitation to be perceptible on the centrifugal compressor sensors, as depicted in Fig. 17(b), which corresponds to the casing vibration as perceived from the rotating frame according to the following equation:

$$
\omega_{\mathrm{s}, \mathrm{R}}^{n_{d}}=\omega_{\mathrm{s}, \mathrm{S}}^{n_{d}}+n_{d} \Omega_{\mathrm{c}}
$$

with $n_{d}=4$ and $\omega_{\mathrm{s}, \mathrm{S}}^{4}=75 \cdot \Omega_{\mathrm{c}}$. On the impeller, this indeed becomes $79 \Omega$, by use of Eq. (3): $\omega_{\mathrm{r} R}^{4}=79 \cdot \Omega_{\mathrm{c}}$. In this same figure, another coincidence between harmonic 77 of the rotating speed $(77 \Omega)$ and the mode with $2 \mathrm{ND}$ is also visible.

Moreover, when $\omega_{\mathrm{r}}^{4}=80 \cdot \Omega_{\mathrm{c}}$ at $t \approx 600 \mathrm{~s}$ one can see some frequencies (black dashed-dotted circles on Fig. 17(b)) with high vibration level every $\Omega$ from $\omega_{\mathrm{r}}^{4}$ (blue dashed-dotted circle). Although these frequencies does not correspond to natural

\footnotetext{
${ }^{1}$ Subscript $\mathrm{S}$ is used for the stationary frame while $\mathrm{R}$ refers to the rotating frame.
} 


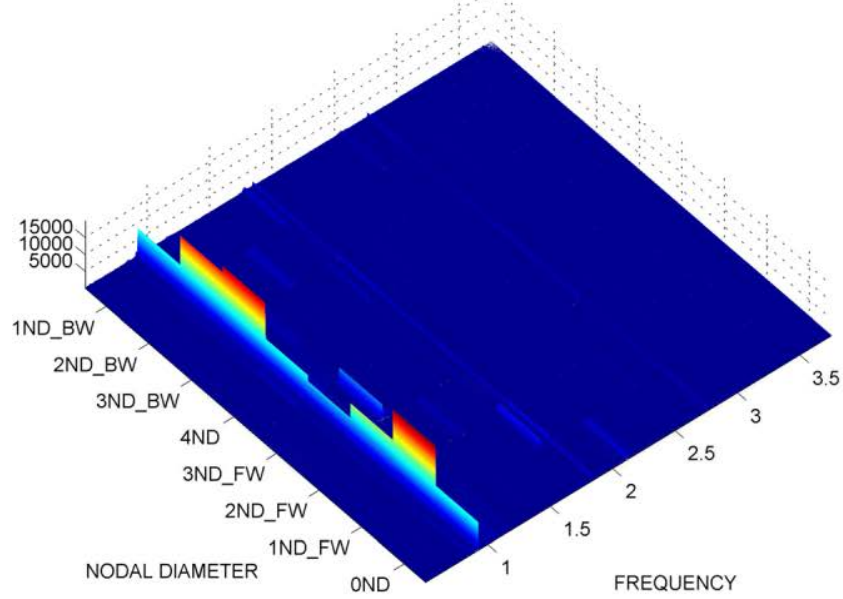

(a)

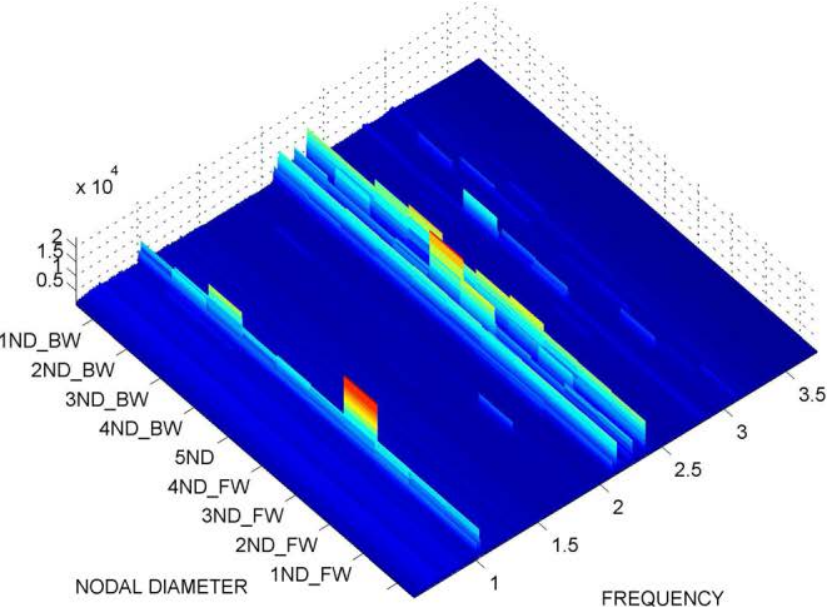

(b)

Fig. 19 2D DFT diagram: eighth burst

impeller frequencies, these frequencies are analogous to sidebands as if vibration at $\omega_{\mathrm{r}}^{4}$ was modulated by rotating frequency [17]. It can be shown that nonlinearity can explain the occurrence of sidebands. A detailed description of sidebands is beyond the scope of this paper and will be discussed in future publications.

During the bursts, speed decreases, thus leading to a sweeping effect of mode frequencies by means of rotating speed harmonic excitation. At the final burst, harmonics $81-87$ of the rotating speed ( $81 \Omega-87 \Omega$ ) coincide with the first family mode with $3 \mathrm{ND}$, as shown in Fig. $18(b)$. On the other hand, the stator's response is dominated by rotating speed harmonic 80 (Fig. 18(a)), which may be interpreted as the frequency of a 3ND vibration of the impeller seen from the stationary frame according to Eq. (3). The spectrograms of Fig. 18 were calculated with a different temporal window than the others spectrograms in order to improve the time resolution despite a lower frequency resolution. Finally, the 2D DFT diagram shown in Fig. 19 highlights the nodal diameter content of the impeller response during the eighth burst. The third nodal diameter forward mode of the predominant first family is observed, along with several modes of higher families. At the same time, the first family modes on the casing with a 1ND forward traveling wave, as well as 2ND and 3ND backward traveling wave, produce the highest amplitudes. The terms forward and backward are correlated with the rotating direction (clockwise). Let us note that on the casing, the modal participation of higher families is imperceptible.

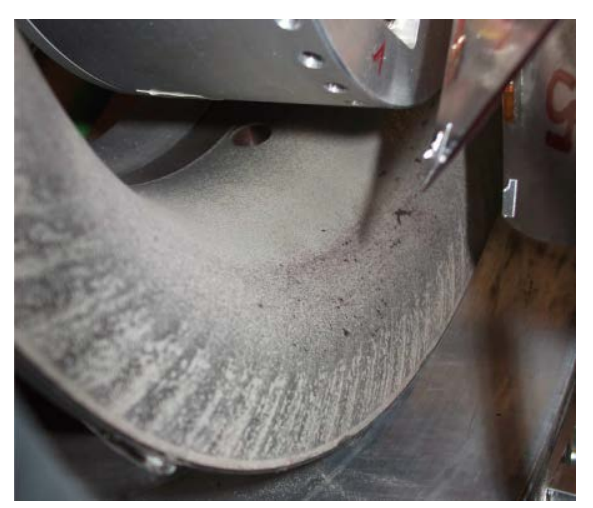

Fig. 20 Wear pattern of the casing

\section{Wear Pattern Analysis}

Measurements of the wear pattern were performed on the trailing edge along the casing circumference, where wear lobes could be seen (Fig. 20). The angular position of the rotor with respect to the casing was recorded simultaneously with the wearing pattern in order to perform a spatial Fourier analysis.

The spatial spectrum of the surface state of the abradable coating is displayed in Fig. 21; the maximum wear depth of $35 \mu \mathrm{m}$ was found for an index number in the spectrum of 83 . As in Ref. [18], this number corresponds to the ratio between the first family mode of the impeller with $3 \mathrm{ND}$ and the rotating speed $\Omega$,

$$
\mathrm{nb}_{\text {lobes }}=\frac{\omega_{\mathrm{r}}}{\Omega}
$$

The previous equation could be considered as a particular case of Eq. (2) where the natural casing frequency is a static shape $\left(\omega_{\mathrm{s}}=0\right)$, and the number of nodal diameter is equal to the number of lobes $\left(n_{d}=\mathrm{nb}_{\text {lobes }}\right)$.

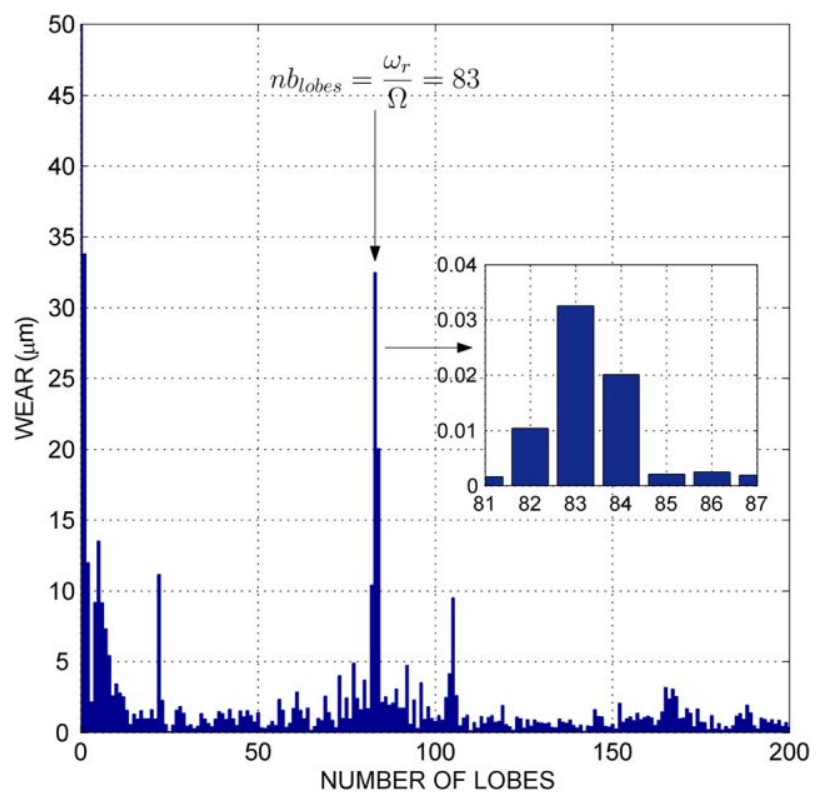

Fig. 21 Spatial spectrum analysis of the wear pattern of the abradable coating 
It can therefore be concluded that this pattern was probably created during the last burst.

\section{Conclusion}

In this paper, experiments have been performed with a rotating centrifugal compressor at constant speed in contact with its casing lined with abradable coating. The contact was initialized by reducing the gap between the two structures. As conclusion, we were summarized the physical behaviors observed during experiments.

Simultaneous dynamical transient events (signal bursts) have been observed on both structures. These bursts were spaced by relatively long time periods and were attributed to contact. During contact, the rotating speed was perturbed and transient of temperatures were measured on the stator. The heating of the casing was first localized and then more distributed as the dynamical behavior increase. The zone of localization could change from one burst to another. This was attributed to abradable wear and/or thermal deformation shape. The dynamic response of the casing was dominated by the first family of modes whereas much more families of modes were involved for the impeller.

In the case of contact both structures were simultaneously excited by rotating speed harmonics. These rotating speed harmonics then coincided with the impeller or casing modes. Such coincidences caused a vibration amplification that led to highlevel transient events (bursts). During these events, speed decreased causing new coincidences with other modes. During a burst, the vibration amplitude may be sufficiently high to wear the casing with a specific pattern. The number of lobes corresponds to the ratio between the excited mode and the rotating speed. The blades were not damaged; however, as the abradable coating mitigated, the severity of contact in order to avoid any degradation on the rotating part. At a different time scale, the thermal behavior appears to raise the system to a higher dynamic vibration level, and this is likely due to the thermal expansion effect.

A low rotating speed causes a high density of its harmonics, which in turn excites both structures when they cross the modes. Further experiments will be performed at higher speeds using the target mode with 3ND. The distance between harmonics of the rotating speed will also be higher, and fewer harmonics will be present in the target frequency range. This revised setup may result in less perturbation to rotating speed harmonics when testing possible modal instability.

\section{Acknowledgment}

This work was partially funded by the French National Research Agency (ANR), within the framework of its COSINUS Technological Research program (IRINA project, ref. ANR 09 COSI 00801 IRINA).

$$
\begin{aligned}
\text { Nomenclature } & \\
\mathrm{DFT} & =\text { discrete Fourier transform } \\
\mathrm{F} & =\text { family of modes } \\
\mathrm{FRF} & =\text { frequency response function } \\
\mathrm{LVDT} & =\text { linear variable differential transformer } \\
n_{d} & =\text { number of nodal diameter } \\
\mathrm{nb}_{\mathrm{lobes}} & =\text { number of wearing lobes }
\end{aligned}
$$

$\mathrm{ND}=$ nodal diameter

PMMA = polymethyl methacrylate

$\mathrm{PZT}=$ lead zirconate titanate piezoelectric material

SLDV $=$ scanning laser Doppler vibrometer

STFT $=$ short-time Fourier transform

$T_{m}=$ time required for the amplitude to decrease by $m$ orders of magnitude

$\mathrm{TC}=$ thermocouples

$\xi=$ damping ratio

$\omega_{\mathrm{r}}^{n_{d}}=$ natural frequency of the impeller with $n_{d}$ diameters

$\omega_{\mathrm{s}}^{n_{d}}=$ natural frequency of the casing with $n_{d}$ diameters

$\Omega=$ rotating speed

$\Omega_{c}=$ critical modal interaction speed

\section{References}

[1] NTSB Bureau of Aviation Safety, 1975, "Aircraft Accident Report: National Airlines, Inc., DC-10-10, N60NA, Near Albuquerque, New Mexico, November 3, 1973," National Transportation Safety Board, Washington, DC, Technical Report NTSB-AAR-75-2, available at: http://lessonslearned.faa.gov/National27/ AccidentInvestigationReport.pdf

[2] Schmiechen, P., 1997, "Travelling Wave Speed Coincidence," Ph.D. thesis, Imperial College of London, London, UK.

[3] Jacquet-Richardet, G., Torkhani, M., Cartraud, P., Thouverez, F., Baranger, T. N., Herran, M., Gibert, C., Baguet, S., Almeida, P., and Peletan, L., 2013 "Rotor to Stator Contacts in Turbomachines. Review and Application," Mech. Syst. Sig. Process., 40(2), pp. 401-420.

[4] Legrand, M., Pierre, C., Cartraud, P., and Lombard, J.-P., 2009, "Two-Dimensional Modeling of an Aircraft Engine Structural Bladed Disk-Casing Modal Interaction," J. Sound Vib., 319(1-2), pp. 366-391.

[5] Williams, R., 2011, "Simulation of Blade Casing Interaction Phenomena in Gas Turbines Resulting From Heavy Tip Rubs Using an Implicit Time Marching Method," ASME Paper No. GT2011-45495.

[6] Meingast, M. B., Batailly, A., Legrand, M., and Ousty, J.-P., 2013. "Investigation of Rotor-Casing Interactions in the Centrifugal Compressor of a Helicopter Engine," ASME Paper No. GT2013-94461.

[7] Padova, C., Barton, J., Dunn, M. G., Manwaring, S., Young, G., Adams, M., and Adams, M., 2005, "Development of an Experimental Capability to Produce Controlled Blade Tip/Shroud Rubs at Engine Speed," ASME J. Turbomach., 127(4), pp. 726-735.

[8] Krajcin, I., and Söffker, D., 2003, "Model-Based Estimation of Contact Forces in Rotating Machines," 4th IMACS Symposium on Mathematical Modeling, Vienna, Austria, Feb. 5-7.

[9] Millecamps, A., Brunel, J.-F., Dufrenoy, P., Garcin, F., and Nucci, M., 2009, "Influence of Thermal Effects During Blade-Casing Contact Experiments," ASME Paper No. DETC2009-86842.

[10] Baiz, S., Fabis, J., Boidin, X., and Desplanques, Y., 2013, "Experimental Investigation of the Blade/Seal Interaction," Proc. Inst. Mech. Eng., Part J, 227(9), pp. 980-995.

[11] Almeida, P., Gibert, C., Leblanc, X., Ousty, J.-P., and Thouverez, F., 2012, "Experimental and Numerical Investigations on a Rotating Centrifugal Compressor," ASME Paper No. GT2012-69760.

[12] Laxalde, D., Gibert, C., and Thouverez, F., 2008, "Experimental and Numerical Investigations of Friction Rings Damping of Blisks," ASME Paper No. GT2008-50862.

[13] Gibert, C., Blanc, L., Almeida, P., Leblanc, X., Ousty, J.-P., Thouverez, F., and Laîné, J.-P., 2012, "Modal Tests and Analysis of a Radial Impeller at Rest: Influence of Surrounding Air on Damping," ASME Paper No. GT2012-69577.

[14] Al-Badour, F., Sunar, M., and Cheded, L., 2011, "Vibration Analysis of Rotating Machinery Using Time-Frequency Analysis and Wavelet Techniques," Mech. Syst. Signal Process., 25(6), pp. 2083-2101.

[15] Flandrin, P., Auger, F., and Chassande-Mottin, E., 2002, "Time-Frequency Reassignment From Principles to Algorithms," Applications in Time Frequency Signal Processing, A. Papandreou-Suppappola, ed., CRC Press, Boca Raton, FL, pp. 179-203.

[16] Gibert, C., Kharyton, V., Thouverez, F., and Jean, P., 2010, "On Forced Response of a Rotating Integrally Bladed Disk: Predictions and Experiments," ASME Paper No. GT2010-23610.

[17] Rzeszucinski, P. J., Sinha, J. K., Edwards, R., Starr, A., and Allen, B., 2012, "Normalised Root Mean Square and Amplitude of Sidebands of Vibration Response as Tools for Gearbox Diagnosis," Strain, 48(6), pp. 445-452.

[18] Millecamps, A., 2010, "Interaction aube-carter: contribution de l'usure de l'abradable et de la thermomécanique sur la dynamique d'aube," Ph.D. thesis, Université de Lille 1, Lille, France. 